\title{
Padronização de alguns parâmetros eletrocardiográficos de animais da espécie Herpailurus yagouaroundi, mantidos em cativeiro
}

\section{Padronization of some electrocardiography parameters of captivity Herpailurus yagouaroundi}

\author{
Sam Goldy Shoyama Oda, ${ }^{*}$ Moacir Leomil Neto, ${ }^{* *}$ José Daniel Luzes Fedullo, ${ }^{* *}$ Ronaldo Jun Yamato, ${ }^{* *}$ \\ Maria Helena Matiko Akao Larsson**
}

\begin{abstract}
Resumo
Vinte e um animais da espécie Herpailurus yagouaroundi, mantidos em cativeiro, foram sedados com uma associação de xilazina (1 a $2 \mathrm{mg} / \mathrm{kg}$ ) e quetamina (10 mg/kg), pela via intramuscular. O traçado eletrocardiográfico foi registrado e padronizado, em todas as derivações, na sensibilidade $1 \mathrm{~cm}=1 \mathrm{mV}$ e na velocidade $25 \mathrm{~mm} / \mathrm{s}$, repetindo-se a derivação DII à velocidade de $50 \mathrm{~mm} / \mathrm{s}$ com mesma sensibilidade. Os resultados obtidos, expressos em média e desvio-padrão, foram FC: 90,952 $\pm 17,293$ (bpm); onda P: 0,030 $\pm 0,006$ (s) X 0,100 $\pm 0,027$ (mV); intervalo PR: 0,089 $\pm 0,014$ (s); complexo QRS: 0,047 $\pm 0,007$ (s) X 1,076 $\pm 0,451$

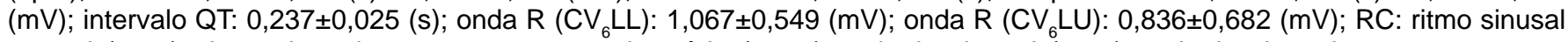
normal (19\%), ritmo sinusal com marcapasso migratório $(4,7 \%)$, arritmia sinusal $(33 \%)$, arritmia sinusal com marcapasso migratório (43\%); eixo elétrico: $+60^{\circ} \mathrm{a}+90^{\circ}(48 \%),+90^{\circ}(4,5 \%),+90^{\circ} \mathrm{a}+120^{\circ}(43 \%),+120^{\circ}(4,5 \%)$; segmento ST: sem desnível $(90 \%)$, supradesnível (10\%); polaridade da onda T (DII): positiva (95\%), negativa (5\%); onda T (V10): negativa (90\%) e interferente $(10 \%)$. Alguns animais da espécie estudada apresentaram valores de amplitude da onda R indicativos de sobrecarga de ventrículo esquerdo, segundo os valores padronizados como normais para felinos domésticos. Exames ecocardiográfico e radiográfico dos mesmos animais demonstraram que o posicionamento cardíaco em relação à caixa torácica era responsável pela variação de amplitude da onda $R$.
\end{abstract}

Palavras-chave: Herpailurus yagouaroundi, felídeos selvagens, eletrocardiograma, cativeiro, padronização.

\begin{abstract}
Twenty one captivity $H$. yagoaroundianesthetized with xilazyn (1 to $2 \mathrm{mg} / \mathrm{kg}$ ) and ketamyn $(10 \mathrm{mg} / \mathrm{kg})$ were used in this study. The ECG tests were registered in all leads with $1 \mathrm{~cm}=1 \mathrm{mV}$ sensibility and $25 \mathrm{~mm} / \mathrm{s}$ speed, repeating the DII lead at the $50 \mathrm{~mm} / \mathrm{s}$ speed with the same sensibility. The results, expressed by mean and standard deviation, were: heart rate $=90,952 \pm 17,293$ (bpm); P wave $=0,030 \pm 0,006(\mathrm{~s}) X 0,100 \pm 0,027(\mathrm{mV})$; PR interval $=0,089 \pm 0,014(\mathrm{~s})$; QRS complex $=0,047 \pm 0,007$ (s) X $1,076 \pm 0,451(\mathrm{mV}) ; \mathrm{QT}$ interval $=0,237 \pm 0,025(\mathrm{~s}) ; \mathrm{R}$ wave $\left(\mathrm{CV}_{6} \mathrm{LL}\right)=1,067 \pm 0,549(\mathrm{mV}) ; \mathrm{R}$ wave $\left(\mathrm{CV}_{6} \mathrm{LU}\right)=0,836 \pm 0,682(\mathrm{mV})$; heart rhythm $=$ normal sinus rhythm $(19 \%)$, sinus rhythm with WPM $(4,7 \%)$, sinus arrythmia $(33 \%)$, sinus arrythmia with WPM $(43 \%)$; electric axis $=+60^{\circ} \mathrm{a}+90^{\circ}(48 \%),+90^{\circ}(4,5 \%),+90^{\circ} \mathrm{a}+120^{\circ}(43 \%),+120^{\circ}(4,5 \%)$; ST segment $=$ no depression or elevation $(90 \%)$, elevation $(10 \%)$; T wave polarity (DII) = positive $(95 \%)$, negative $(5 \%)$; V10 (T wave) = negative $(90 \%)$, and interferent $(10 \%)$. Some of the studied animals presented $\mathrm{R}$ wave amplitude that was indicative of left ventricle overload, according to the patterns for normal domestic cats. Echocardiographic and radiographic exams revealed different heart position in the thorax, explaining the $\mathrm{R}$ wave amplitude variation.
\end{abstract}

Keywords: Herpailurus yagouaroundi, wild cats, electrocardiography, captivity, padronization.

\section{Introdução}

A destruição do hábitat dos felinos selvagens por inúmeros fatores, tais como o avanço das fronteiras agrícolas, a exploração de minérios e madeira, o povoamento e a construção de represas para abastecer usinas hidrelétricas
(OLIVEIRA, 1994), somados à caça ilegal são suficientes para explicar o declínio da sua população.

Considerando que todas as espécies de felinos selvagens brasileiros encontram-se ameaçadas de extinção, surgiu um interesse muito grande por parte dos Jardins Zoológicos, tanto

\footnotetext{
* Autônomo

** Faculdade de Medicina Veterinária e Zootecnia - USP; Avenida Prof. Dr. Orlando Marques de Paiva, 87 - CEP $05508-000$ - São Paulo, SP. Tel. (011) 30911262 - E-mail: akaolar@usp.br.

*** Fundação Parque Zoológico de São Paulo.
} 
nacionais quanto internacionais, no que diz respeito à manutenção e reprodução em cativeiro (OLIVEIRA e CASSARO, 1999) das mesmas.

Problemas como a ocorrência de doenças, condições inadequadas em que são mantidos, número reduzido de animais em cativeiro e elevado coeficiente de consangüinidade constituem fatores que dificultam a adaptação, manutenção e reprodução em condições de cativeiro.

Manejo intenso, como a translocação de indivíduos de uma população para outra, proporcionando uma difusão da genética de diferentes populações de uma mesma espécie, é uma forma de viabilizar a sobrevivência, a longo prazo, de pequenas populações.

Frente a tantas dificuldades, alguns zoológicos têm-se empenhado em realizar programas de reprodução para determinadas espécies de gatos tropicais, em especial a Fundação Parque Zoológico de São Paulo, mentor do Projeto Internacional de Reprodução e Conservação de Felinos (OLIVEIRA, 1994).

A padronização dos parâmetros eletrocardiográficos da espécie Herpailurus yagouaroundi, objetivo do presente trabalho, visa contribuir com o referido projeto de reprodução e conservação de felinos, proporcionando valores de referência para melhor conhecimento da referida espécie.

\section{Material e métodos}

Vinte e um indivíduos da espécie Herpailurus yagouaroundi (gato mourisco), mantidos em cativeiro, foram avaliados por meio de exame eletrocardiográfico.

Os animais, imunizados com vacina tríplice contra panleucopenia, rinotraqueíte e calicivirose (vacina Fel-OVanPCT-lab Fort-Dodge) e mantidos em jaulas coletivas, recebiam alimentação diferenciada, a saber: músculo bovino, coração bovino, sardinha, suplemento vitamínico e mineral, sendo que a alimentação às terças e quintas-feiras era constituída, apenas, de pintos de um dia.

Para a realização do exame os animais foram anestesiados com xilazina e quetamina nas doses, respectivamente, de 1 a $2 \mathrm{mg} / \mathrm{kg}$ e $10 \mathrm{mg} / \mathrm{kg}$, com auxílio de caixas de contenção.

O exame eletrocardiográfico foi realizado utilizando-se eletrocardiógrafo ECAFIX modelo ECG 6. Para tanto, eram posicionados em decúbito lateral direito, sobre tapete de borracha para evitar interferências, eletrodos eram fixados à pele, por meio de clipes do tipo "jacaré", aplicando-se, em seguida, álcool líquido nos pontos de fixação para um melhor contato com o corpo do animal.

O traçado eletrocardiográfico foi registrado e padronizado da seguinte forma: traçado de todas as derivações em sensibilidade $1 \mathrm{~cm}=1 \mathrm{mV}$ e em velocidade $25 \mathrm{~mm} / \mathrm{s}$ (TILLEY, 1992; KITLESON \& KIENLE, 1998; MILLER et al., 1999), repetindo-se a derivação DII à velocidade de $50 \mathrm{~mm} / \mathrm{s}$ com sensibilidade $1 \mathrm{~cm}=1 \mathrm{mV}$.

Para a obtenção do registro das derivações do plano frontal DI, DII e DIII, que são as derivações bipolares, e aVR, aVL e aVF, que são as derivações unipolares aumentadas, os eletrodos dos membros torácicos foram fixados logo acima da região do olécrano e os eletrodos dos membros pélvicos logo acima da região da patela (TILLEY, 1992; KITLESON \& KIENLE, 1998; MILLER et al., 1999).

Realizaram-se também as derivações unipolares précordiais, as quais medem o potencial elétrico do coração no plano transversal: $C_{5} R L, C_{6} L L, C_{6} L U$ e $V_{10}$ (TILLEY, 1992). Os valores obtidos foram submetidos à análise estatística descritiva, para o cálculo do valor médio e do desvio-padrão.

A análise dos resultados foi realizada utilizando-se o teste "Two sample T-test and Confidence Interval" do Minitab 14 for Windows para avaliar o grau de significância das diferenças entre as médias obtidas e os valores encontrados em trabalhos publicados anteriormente. Com adoção de $\mathrm{a}=0,05$, foram considerados estatisticamente semelhantes os valores médios que, após serem comparados, apresentaram p>0,05.

\section{Resultados e discussão}

Os resultados dos parâmetros eletrocardiográficos avaliados (freqüência cardíaca, duração e amplitude da onda $P$, intervalo PR, duração e amplitude do complexo QRS, intervalo QT, onda $\mathrm{R}$ em $\mathrm{CV}_{6} \mathrm{LL}$ e $\mathrm{CV}_{6} \mathrm{LU}$, ritmo cardíaco, eixo elétrico, segmento ST, polaridade da onda T em DII e V10) acham-se consubstanciados nas Tabelas 1 e 2.

Os parâmetros eletrocardiográficos qualitativos, a saber: ritmo, eixo, segmento ST, polaridade da onda T em DIl e V10 são apresentados sob a forma descritiva (percentual). Ritmo cardíaco: ritmo sinusal normal (19\%), ritmo sinusal com marca-passo migratório $(4,7 \%)$, arritmia sinusal (33\%), arritmia sinusal com marca-passo migratório (43\%); eixo elétrico: $+60^{\circ} \mathrm{a}+90^{\circ}(48 \%),+90^{\circ}(4,5 \%),+90^{\circ} \mathrm{a}+120^{\circ}(43 \%)$, $+120^{\circ}(4,5 \%)$; segmento ST: sem desnível (90\%), supradesnível (10\%); polaridade da onda T (DII): positiva (95\%), negativa (5\%); onda T (V10): negativa (90\%), interferente (10\%).

A amplitude da onda R (em DII), apresentada por alguns animais da espécie, poderia ser indicativa de aumento de ventrículo esquerdo se comparada com os parâmetros, ditos normais ( $\mathrm{R} £ 0,9 \mathrm{mV}$ ), para $F$. cati (gatos domésticos); no entanto, o exame ecocardiográfico desses animais não revelou nenhuma alteração de tamanho das cavidades cardíacas.

Com o intuito de encontrar uma explicação plausível para tal aumento de onda $R$, foram realizadas radiografias, tanto de animais que apresentaram onda $R$ dentro dos parâmetros de normalidade, para gatos domésticos, como daqueles que apresentaram onda $R$ aumentada. $A$ análise das radiografias revelou que os animais que apresentaram onda $R>0,9 \mathrm{mV}$ tinham o coração mais horizontalizado em relação à caixa torácica, do que os animais que apresentavam amplitude de onda $\mathrm{R}$ dentro dos limites de normalidade, para gatos domésticos. Assim sendo, uma possível explicação para tal achado eletrocardiográfico seria o posicionamento do coração dentro da cavidade torácica, isto é, coração mais horizontalizado proporcionando uma onda $\mathrm{R}$ de maior amplitude, sem que isso signifique aumento ventricular esquerdo, e coração mais verticalizado proporcionando uma onda $\mathrm{R}$ de menor amplitude.

A influência originada pela anestesia sobre os parâmetros eletrocardiográficos analisados deve ser levada em conta. 
Tabela 1: Média e desvio-padrão de freqüência cardíaca, duração e amplitude da onda $P$, intervalo PR, duração e amplitude do complexo QRS, tipos de ritmo cardíaco e eixo cardíaco de felídeos da espécie Herpailurus yagouaroundi anestesiados com xilazina e quetamina - São Paulo, 2005

\begin{tabular}{ccccccccc}
\hline $\mathrm{N}^{\circ}$ & FC & RíTMO & ElXO & $\mathrm{P}(\mathrm{s})$ & $\mathrm{P}(\mathrm{mV})$ & $\mathrm{PR}(\mathrm{s})$ & QRS $(\mathrm{s})$ & QRS $(\mathrm{mV})$ \\
\hline 1 & 100 & AS & $+60^{\circ} \mathrm{a}+90^{\circ}$ & 0,03 & 0,10 & 0,07 & 0,06 & 1,45 \\
\hline 2 & 90 & AS MPM & $+90^{\circ} \mathrm{a}+120^{\circ}$ & 0,03 & 0,10 & 0,09 & 0,05 & 1,10 \\
\hline 3 & 80 & AS MPM & $+60^{\circ} \mathrm{a}+90^{\circ}$ & 0,03 & 0,10 & 0,10 & 0,04 & 1,00 \\
\hline 4 & 100 & AS MPM & $+90^{\circ} \mathrm{a}+120^{\circ}$ & 0,03 & 0,10 & 0,10 & 0,04 & 0,80 \\
\hline 5 & 80 & AS MPM & $+60^{\circ} \mathrm{a}+90^{\circ}$ & 0,03 & 0,10 & 0,09 & 0,04 & 1,50 \\
\hline 6 & 60 & AS & $+60^{\circ} \mathrm{a}+90^{\circ}$ & 0,02 & 0,10 & 0,09 & 0,05 & 1,45 \\
\hline 7 & 80 & RSN & $+90^{\circ}$ & 0,04 & 0,10 & 0,11 & 0,06 & 0,90 \\
\hline 8 & 100 & AS MPM & $+60^{\circ} \mathrm{a}+90^{\circ}$ & 0,03 & 0,10 & 0,08 & 0,05 & 1,90 \\
\hline 9 & 100 & AS & $+90^{\circ} \mathrm{a}+120^{\circ}$ & 0,02 & 0,10 & 0,08 & 0,05 & 2,10 \\
\hline 10 & 100 & RSN & $+60^{\circ} \mathrm{a}+90^{\circ}$ & 0,03 & 0,10 & 0,11 & 0,04 & 0,90 \\
\hline 11 & 80 & AS MPM & $+90^{\circ} \mathrm{a}+120^{\circ}$ & 0,02 & 0,10 & 0,08 & 0,04 & 1,20 \\
\hline 12 & 80 & AS MPM & $+60^{\circ} \mathrm{a}+90^{\circ}$ & 0,03 & 0,10 & 0,10 & 0,04 & 0,50 \\
\hline 13 & 80 & AS & $+90^{\circ} \mathrm{a}+120^{\circ}$ & 0,03 & 0,10 & 0,11 & 0,05 & 1,40 \\
\hline 14 & 80 & AS MPM & $+120^{\circ}$ & 0,04 & 0,20 & 0,10 & 0,04 & 0,30 \\
\hline 15 & 80 & RS MPM & $+60^{\circ} \mathrm{a}+90^{\circ}$ & 0,03 & 0,10 & 0,11 & 0,05 & 0,90 \\
\hline 16 & 80 & AS MPM & $+60^{\circ} \mathrm{a}+90^{\circ}$ & 0,03 & 0,05 & 0,08 & 0,05 & 1,00 \\
\hline 17 & 80 & AS & $+90^{\circ} \mathrm{a}+120^{\circ}$ & 0,02 & 0,05 & 0,08 & 0,06 & 1,00 \\
\hline 18 & 100 & AS & $+60^{\circ} \mathrm{a}+90^{\circ}$ & 0,03 & 0,10 & 0,07 & 0,04 & 0,90 \\
\hline 19 & 100 & RSN & $+90^{\circ} \mathrm{a}+120^{\circ}$ & 0,04 & 0,10 & 0,08 & 0,04 & 1,00 \\
\hline 20 & 140 & AS & $+90^{\circ} \mathrm{a}+120^{\circ}$ & 0,04 & 0,10 & 0,07 & 0,05 & 1,00 \\
\hline 21 & 120 & RSN & $+90^{\circ} \mathrm{a}+120^{\circ}$ & 0,03 & 0,10 & 0,07 & 0,05 & 0,30 \\
\hline MEDIA & 90,95 & & 0,03 & 0,10 & 0,09 & 0,05 & 1,08 \\
\hline DP & 17,29 & & 0,01 & 0,03 & 0,01 & 0,01 & 0,45 \\
\hline & & & & & & & \\
\hline
\end{tabular}

Tabela 2: Média e desvio-padrão do intervalo QT, amplitude da onda $R$ em $C V_{6} L L$ e $C V_{6} L U$,

\begin{tabular}{|c|c|c|c|c|c|c|}
\hline № & ST & $\mathrm{T}$ & QT (s) & $\begin{array}{c}\mathrm{CV}_{6} \mathrm{LL} \\
(\mathrm{R} \text { em mV) }\end{array}$ & $\begin{array}{c}\mathrm{CV}_{6} \mathrm{LU} \\
(\mathrm{R} \text { em mV) }\end{array}$ & $\begin{array}{c}\mathrm{V}_{10} \\
\text { (onda T) }\end{array}$ \\
\hline 1 & sem desnível & positiva & 0,20 & 0,40 & 0,40 & T negativa \\
\hline 2 & sem desnível & positiva & 0,26 & 1,50 & 0,90 & T negativa \\
\hline 3 & sem desnível & positiva & 0,24 & 2,10 & 1,00 & interferente \\
\hline 4 & sem desnível & positiva & 0,22 & 1,20 & 1,00 & T negativa \\
\hline 5 & sem desnível & positiva & 0,24 & 1,30 & 0,30 & interferente \\
\hline 6 & sem desnível & positiva & 0,28 & 0,55 & 1,80 & T negativa \\
\hline 7 & supradesnível & positiva & 0,25 & 0,45 & 0,20 & T negativa \\
\hline 8 & sem desnível & positiva & 0,20 & 1,20 & 2,90 & T negativa \\
\hline 9 & sem desnível & positiva & 0,21 & 0,90 & 0,30 & T negativa \\
\hline 10 & sem desnível & positiva & 0,22 & 0,40 & 0,15 & T negativa \\
\hline 11 & sem desnível & positiva & 0,22 & 0,70 & 0,60 & T negativa \\
\hline 12 & sem desnível & positiva & 0,24 & 0,90 & 0,70 & T negativa \\
\hline 13 & sem desnível & positiva & 0,30 & 1,60 & 0,70 & T negativa \\
\hline 14 & sem desnível & positiva & 0,24 & 0,80 & 0,30 & T negativa \\
\hline 15 & sem desnível & positiva & 0,24 & 1,50 & 1,50 & T negativa \\
\hline 16 & sem desnível & positiva & 0,26 & 2,20 & 1,40 & T negativa \\
\hline 17 & sem desnível & negativa & 0,24 & 1,10 & 1,00 & T negativa \\
\hline 18 & sem desnível & positiva & 0,24 & 0,30 & 0,60 & T negativa \\
\hline 19 & sem desnível & positiva & 0,22 & 1,40 & 1,40 & T negativa \\
\hline 20 & supradesnível & positiva & 0,24 & 1,40 & 0,40 & T negativa \\
\hline 21 & sem desnível & positiva & 0,21 & 0,50 & 0,00 & T negativa \\
\hline MÉDIA & & & 0,24 & 1,07 & 0,84 & \\
\hline DP & & & 0,02 & 0,55 & 0,68 & \\
\hline
\end{tabular}

\section{desnível do segmento ST, polaridade da onda T e polaridade da onda $\mathrm{T}$ em $\mathrm{V}_{10}$
de felídeos da espécie Herpailurus yagouaroundi anestesiados com xilazina e \\ Tesnível do segmento ST, polaridade da onda T e polaridade da onda $\mathrm{T}$ em $\mathrm{V}_{10}$,
de felídeos da espécie Herpailurus yagouaroundi anestesiados com xilazina e quetamina - São Paulo, 2005}

FC: freqüência cardíaca; RSN: ritmo sinusal normal; RS MPM: ritmo sinusal com marca-passo migratório; AS: arritmia sinusal; AS MPM: arritmia sinusal com marca-passo migratório; EIXO eixo elétrico; DP: desvio-padrão 
A xilazina é um fármaco do grupo dos agentes $\mathrm{a}_{2}$-agonistas, os quais causam sedação dose-dependente. $A$ depressão dose-dependente é decorrente da estimulação de receptores $a_{2}$-adrenérgicos tanto no sistema nervoso central quanto no sistema nervoso periférico. A xilazina promove também a diminuição da liberação de norepinefrina central e periférica, verificando-se diminuição da atividade simpática (Fantoni e Cortopassi, 2002).

Diminuição da freqüência cardíaca, bloqueios atrioventriculares de 1ㅇ, ㄴo e até 3 o grau, redução do débito cardíaco e indução do aumento inicial da pressão arterial, seguido de hipotensão duradoura, são efeitos cardiovasculares produzidos pela xilazina (Fantoni e Cortopassi, 2002).

A quetamina, por sua vez, é um fármaco do grupo dos anestésicos dissociativos, de efeito predominantemente simpatomimético, que tem como principais mecanismos de ação: interação com receptores do tipo $\mathrm{N}$-metil-D-aspartato (NMDA) reduzindo, seletivamente, a excitação produzida pelos aminoácidos excitatórios; bloqueio dos receptores muscarínicos dos neurônios centrais, podendo potencializar os efeitos inibitórios do GABA; bloqueio do processo de transporte neuronal da serotonina, dopamina e noradrenalina, potencializando os efeitos dessas catecolaminas por bloquearem a recaptação desses neurotransmissores (Fantoni e Cortopassi, 2002).

Segundo Fantoni e Cortopassi (2002), aumentos da freqüência cardíaca, do débito cardíaco, da pressão arterial média, da pressão na artéria pulmonar, da pressão venosa central, além de efeito inotrópico positivo sobre a musculatura cardíaca podem ser decorrentes da ação da quetamina sobre o sistema cardiovascular. Já o efeito sobre o ritmo cardíaco é controverso.

O efeito estimulante, produzido pela quetamina sobre o sistema cardiovascular, pode ser prevenido ou diminuído pela pré-administração de $\mathrm{a}_{2}$-agonistas, devido à diminuição da atividade simpática gerada (Fantoni e Cortopassi, 2002).

\section{Referências}

FANTONI, D. T.; CORTOPASSI, S. R. G. In: Gatos. São Paulo: Roca, 2002, p.151-173. Anestesia em Cãese

KITLESON, M. D.; KIENLE, R. D. Electrocardiography: basic concepts, diagnosis of chambers enlargement, and intraventricular conduction disturbances. In: Small animal cardiovascular medicine. St Louis: Mosby, 1998, p. $72-94$.

MILLER, M. S.; TILLEY, L. P.; SMITH JR., F. W.; FOX, P. R. Electrocardiography. In: FOX, P. R.; SISSON, D.; MOISE, S., Canine and feline cardiology. 2. ed. Philadelphia: W. B. Saunders, 1999, p. 67-105.
Em cães, a xilazina é amplamente utilizada em associação à quetamina para a realização de procedimentos cirúrgicos de pequeno porte. No entanto, nas associações convencionais, normalmente empregam-se doses elevadas de xilazina, cujos efeitos cardiovasculares adversos nem sempre são contrabalanceados pela ação simpatomimética da quetamina (Fantoni e Cortopassi, 2002).

Levando-se em conta os aspectos cardiovasculares dos fármacos em questão, é clara a interferência que pode vir a ocorrer sobre os parâmetros eletrocardiográficos, principalmente freqüência e ritmo cardíacos, e pressóricos. No entanto, esta interferência pode estar sendo minimizada, já que os efeitos sobre o sistema cardiovascular dos diferentes fármacos são antagônicos.

Porém, com relação à anestesia, o principal fator que minimiza a importância de sua interferência na padronização dos parâmetros eletrocardiográficos em questão é o fato de a anestesia ser imprescindível para a realização de um exame eletrocardiográfico nesses espécimes.

\section{Conclusões}

Os resultados obtidos, por meio da metodologia utilizada, permitem afirmar que:

os parâmetros eletrocardiográficos de $H$. yagouaroundi são diversos daqueles da espécie $F$. catus, exceto a duração da onda P; na espécie estudada, predominantemente, o ritmo cardíaco é a arritmia sinusal com marca-passo migratório, o eixo cardíaco entre $+60^{\circ} \mathrm{a}+90^{\circ}$, o segmento ST sem desnível, a polaridade da onda T negativa em V10 e positiva em DII.

Em suma, apesar da semelhança fenotípica entre as espécies $H$. yagouaroundi e $F$. catus (gato doméstico) os parâmetros anteriormente estabelecidos para esta espécie não podem ser utilizados para outros felinos de pequeno porte.

OLIVEIRA, T. G. Neotropical Cats Ecology and Conservation. São Luís: EDUFMA, 1994, 220 p.

OLIVEIRA, T. G., CASSARO, K. Guia de identificação dos felinos brasileiros. 2.ed. São Paulo: Sociedade de Zoológicos do Brasil, 1999, $60 \mathrm{p}$.

TILLEY, L. P. General principles of eletrocardiography. In: Essentials of canine and feline electrocardiography. 3. ed. Philadelphia: Lea \& Febiger, p. 21-55, 1992. 